\title{
miRNA-141 is a novel regulator of BMP-2-mediated calcification in aortic stenosis
}

\author{
Bobby Yanagawa, MD, PhD, Fina Lovren, PhD, Yi Pan, MD, Vinay Garg, BHSc, Adrian Quan, MPhil, \\ Gilbert Tang, MD, Krishna K. Singh, PhD, Praphulla C. Shukla, PhD, Nikhil P. Kalra, BSc, \\ Mark D. Peterson, MD, PhD, and Subodh Verma, MD, PhD
}

\begin{abstract}
Objective: Bone morphogenetic protein-2 (BMP-2) is a major regulator of aortic valve calcification. MicroRNAs (miRNAs) are essential post-transcriptional modulators of gene expression and miRNA-141 is a known repressor of BMP-2-mediated osteogenesis. We hypothesized that miRNA-141 is a key regulator of aortic valve calcification.
\end{abstract}

\begin{abstract}
Methods: Porcine valvular interstitial cells were isolated, transfected with miRNA-141 or control, and stimulated with transforming growth factor- $\beta$. The BMP-2, extracellular signal-regulated kinase $1 / 2$, and runt-related transcription factor 2 levels were determined by immunoblotting and reverse transcriptase polymerase chain reaction. To determine the role of miRNA-141 in bicuspid aortic valve disease, human bicuspid $(\mathrm{n}=19)$ and tricuspid $(\mathrm{n}=17)$ aortic valve leaflets obtained intraoperatively were submitted for GenoExplorer human microRNA array, immunoblotting, and histologic and immunohistochemical analyses.
\end{abstract}

Results: Stimulation of porcine aortic valvular interstitial cells with transforming growth factor- $\beta$ induced morphologic alterations consistent with myofibroblastic transformation, BMP-2 signaling, and calcification. Transfection with miRNA-141 restored transforming growth factor- $\beta$-induced valvular interstitial cell activation, BMP-2 signaling, and alkaline phosphatase activity $(3.55 \pm 0.18$ vs $4.01 \pm 0.21, P<.05)$, suggesting upstream regulation by miRNA-141. miRNA microarray demonstrated differential expression of 35 of 1583 miRNA sequences in the bicuspid versus tricuspid aortic valve leaflets, with a 14.5-fold decrease in miRNA-141 in the bicuspid versus tricuspid leaflets $(P<.05)$. This was associated with significantly increased BMP-2 protein expression in bicuspid aortic valve compared with the tricuspid aortic valve leaflets $(P<.001)$.

Conclusions: We report a completely novel role of miRNA-141 as a regulator of BMP-2-dependent aortic valvular calcification and demonstrate marked attenuation of miRNA-141 expression in patients with bicuspid aortic valve-associated aortic stenosis. Therapeutic targeting of miRNA-141 could serve as a novel strategy to limit progressive calcification in aortic stenosis. (J Thorac Cardiovasc Surg 2012;144:256-62)

\section{Supplemental material is available online.}

Aortic valve stenosis, once conceptualized as degenerative calcification, is now appreciated to be a complex and active process characterized by endothelial injury, inflammatory infiltration, angiogenesis, valvular interstitial cell-tomyofibroblast transformation, calcification, and matrix turnover. ${ }^{1}$

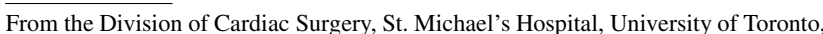
Toronto, Ontario, Canada.

Disclosures: Authors have nothing to disclose with regard to commercial support. Received for publication July 10, 2011; revisions received Oct 4, 2011; accepted for publication Oct 26, 2011; available ahead of print Feb 16, 2012.

Address for reprints: Subodh Verma, MD, PhD, Division of Cardiac Surgery, St Michael's Hospital, Suite 8-003F, Bond Wing, 30 Bond Street, Toronto, ON M5B 1W8 Canada (E-mail: subodh.verma@sympatico.ca).

$0022-5223 / \$ 36.00$

Copyright (c) 2012 by The American Association for Thoracic Surgery doi:10.1016/j.jtcvs.2011.10.097
Valvular calcification is the defining feature of aortic stenosis (AS), with accumulating evidence for heterotopic ossification. $^{2}$ Human aortic valvular interstitial cells (VICs) isolated from stenotic aortic valves express osteogenic signals, notably the bone morphogenetic proteins (BMPs) of the transforming growth factor- $\beta$ (TGF- $\beta$ ) family. ${ }^{2,3}$ The BMP family of proteins is a regulator of cell growth, apoptosis, and differentiation of mesechmymal cells into osteoblasts and chondrocytes. ${ }^{4}$ These markers are expressed during active mineralization and osteoblast differentiation and tend to co-localize to areas of calcification. ${ }^{5,6}$ Of these, BMP-2 is a potent stimulant of valvular calcification in vivo and in vitro. ${ }^{3}$ The downstream elements of BMP-2 include the transcription factors runt-related transcription factor 2 (RunX2) and distal-less homeodomain 5 (Dlx5). ${ }^{7,8}$ RunX2 is a transcription factor involved in the regulation of fibroblast differentiation and bone formation. ${ }^{9}$ There are several downstream protein targets, such as osteopontin, an important matrix glycoprotein of bone, that is associated with calcification and atherosclerosis in human arteries and in a variety of human tissues. ${ }^{10}$ 


$$
\begin{aligned}
& \text { Abbreviations and Acronyms } \\
& \begin{aligned}
\text { AS } & =\text { aortic stenosis } \\
\text { BMP-2 } & =\text { bone morphogenetic protein- } 2 \\
\text { BAV } & =\text { bicuspid aortic valve } \\
\text { Dlx } 5 & =\text { distal-less homeodomain } 5 \\
\text { miR } & =\text { microRNA or miRNA } \\
\text { RunX2 } & =\text { runt-related transcription factor- } 2 \\
\alpha-\mathrm{SMA} & =\alpha \text {-smooth muscle actin } \\
\text { TAV } & =\text { tricuspid aortic valve } \\
\text { TGF- } \beta & =\text { transforming growth factor }-\beta \\
\text { VIC } & =\text { valvular interstitial cell }
\end{aligned}
\end{aligned}
$$

MicroRNAs (miRNAs) are evolutionarily conserved 22-mer RNA molecules that are emerging as essential post-transcriptional modulators of gene expression, which coordinate and integrate multiple regulatory pathways involved in normal and abnormal development. Current predictions suggest that miRNAs might regulate expression of approximately $30 \%$ of all protein-coding genes at baseline that increases with stress or injury. ${ }^{11}$ miRNA-141 is a key regulator of BMP-2-mediated osteogenesis but its role in valvular calcification is unknown. ${ }^{12}$

In the present study, we examined the role of miRNA-141 on regulation of BMP-2-dependent calcification in cultured VICs. Bicuspid aortic valve (BAV) is a common congenital cardiac anomaly, with an estimated incidence of $1 \%$ to $2 \%{ }^{13}$ It is responsible for the greatest burden of aortic valve disease in patients younger than 70 years old in North America. To determine whether altered expression of miRNA-141 is associated with early calcification in BAVs, we performed microRNA profiling. We identify miRNA-141 downregulation in human BAV versus tricuspid aortic valve (TAV) leaflets, which is associated with increased expression of BMP-2 in the areas of valvular calcification.

\section{METHODS}

\section{VIC Culture and Transient Transfection}

Aortic VICs were harvested from 10 porcine hearts (4-6 months old), as previously described ${ }^{14}$ In brief, aortic valves were excised with care not to introduce any nonleaflet tissue. The leaflets were gently scraped on the aortic and ventricular aspects to remove the endothelial layer, and the tissues were then sectioned into pieces approximately $2 \times 2 \mathrm{~mm}$ and explant cultured in growth medium (M199 medium with penicillin G, streptomycin, amphotericin B, and $10 \%$ fetal bovine serum) in an incubator supplied with $5 \%$ carbon dioxide for 10 days. The cells growing from the explants were subcultured, and the cells from passages 3 to 4 were used for additional study.

Monolayers of VICs $\left(7.5 \times 10^{4}\right.$ cells $\left./ \mathrm{cm}^{2}\right)$ were transfected with $2 \mathrm{nM}$ of the miRIDIAN mimic microRNA-141 or a scrambled control (ThermoScientific, Waltham, Mass) using the Dharmafect transfection reagent (Dharmacon, Lafayette, Colo), according to the manufacturer's recommendations. The transfected cells were further cultured with or without $5 \mathrm{ng} / \mathrm{mL}$ TGF- $\beta$ (R\&D Systems, Minneapolis, Minn) for 6 to 12 days in the low serum medium ( $1 \%$ fetal bovine serum supplemented). The medium was changed every 3 days. The cells were harvested, and the total protein and RNA were extracted for analysis.

\section{Study Population and Data Collection}

Patients undergoing elective aortic valve replacement for AS at St Michael's Hospital from June 2010 to June 2011 were enrolled. The aortic valve leaflets were obtained intraoperatively from patients with congenital BAVs $(n=19)$ and TAVs $(n=17)$ at valve replacement. The leaflets were sectioned into 2 equal parts; 1 part was flash frozen in liquid nitrogen and submitted for RNA or protein extraction, and 1 part was fixed in $10 \%$ formalin and embedded in paraffin.

The patients were identified as having congenital, and not functional, BAV using clinical criteria according to the following characteristics: (1) age younger than 65 years at surgery, (2) a positive history of murmur since adolescence, (3) a positive family history of BAV, (4) valve leaflet and sinus asymmetry on the echocardiogram and intraoperatively, and (5) an association with ascending aorta or aortic root dilation. Patients deemed questionable for having congenital BAV were excluded. Additional exclusion criteria included a nonstenotic, primary aortic valve regurgitant phenotype, infective endocarditis, or rheumatic aortic valvulopathy. The St Michael's Hospital institutional review board approved the study, and all patients provided written informed consent.

\section{miRNA Array}

miRNA was isolated using the miRNeasy kit (Qiagen, Hilden, Germany) according to the manufacturer's instructions. For miRNA microarray analysis, total RNA was directly labeled with biotin and hybridized to the GenoExplorer microRNA human array containing 1583 human miRNA probes (Genosensor, Tempe, Ariz), and the fluorescent signals were then scanned using a GenePix 4000b Biochip. The average of 3 mean fluorescence signal intensities for each miRNA probe was normalized to that for tRNAmet. Precursor miRNAs detected at twofold greater than the background were considered to be expressed. Data were analyzed using GenePix, version 5.0, software (provided by GenoSensor, Temple, Ariz). The complete annotated miRNA microarray raw and normalized dataset is available at the National Institutes of Health/National Center for Biotechnology Information, Gene Expression Omnibus (accession number GSE30197).

\section{Histologic Examination, Terminal Deoxynucleotidyl Transferase-Mediated Deoxyuridine Triphosphate Nick End Labeling, and Immunohistochemistry}

Formalin-fixed and paraffin-embedded tissues were analyzed using hematoxylin-eosin, Masson's trichrome, and von Kossa histochemical analysis. The tissues were also analyzed with immunohistochemistry for BMP-2 (Abcam, Cambridge, Mass) and $\alpha$-smooth muscle actin $(\alpha$-SMA). In brief, the sections were washed in Tris-buffered saline/ $0.1 \%$ Tween-20 then blocked in 5\% milk. The sections were incubated in primary antibody (1:5000) overnight, washed, and then incubated in biotinylated secondary antibody $(1: 10,000)$ for 30 minutes. The sections were treated with Vectastain $\mathrm{ABC}$ reagent (Vector, Burlingame, Calif), then $\mathrm{DAB}$, and counterstained with hematoxylin. ImageJ software (available from http://rsbweb.nih.gov/ij) was used for signal quantitation.

\section{Real-Time Polymerase Chain Reaction for Detection of MicroRNA-141, BMP-2, and RunX2 Expression}

VIC microRNAs were extracted using the miRNeasy Mini Kit (Qiagen, ON, Canada) and reverse transcribed with the RT2 miRNA First Strand Kit (SA Biosciences, Frederick, MD), as per the manufacturer's instructions. Real-time polymerase chain reaction was performed on the StepOnePlus Real-Time Polymerase Chain Reaction System (Applied Biosystems, Foster City, Calif) using the RT2 SybrGreen quantitative PCR Mastermix 
(SA Biosciences) combined with either microRNA-141 or RNU6 primers (SA Biosciences), BMP-2 (forward 5'-TCTGCGGTCTCCTAAAGGTC- ${ }^{\prime}$ and reverse 5'-GGAAGCAGCAACGCTAGAAG-3'), RunX2 (forward $5^{\prime}$-CACACCTACCTGCCACCAC- $3^{\prime}$ and reverse $5^{\prime}$-TCCTGACGAGGT GCCATAG-3'), and glyceraldehyde-3-phosphate dehydrogenase (forward 5'-CACCAGGGCTGCTTTTAACTCTGGTA- $3^{\prime}$ and reverse $5^{\prime}$-CCTTGA CGGTGCCATGGAATTGC- $3^{\prime}$ ) primers. Gene expression data were analyzed using Pfaffl's relative expression software (REST-MSC, version 2, available from http://www.wzw.tum.de/gene-quantification/).

\section{Alkaline Phosphatase Activity in VICs}

The alkaline phosphatase colorimetric assay kit (Abcam, Cambridge, UK) was used to measure alkaline phosphatase activity in the cell lysate collected from the transfected or control VICs after stimulation with TGF- $\beta$ for 6 days.

\section{Western Blot}

For detection of BMP-2 expression, proteins were isolated from the human aortic valve leaflets, which were mechanically disrupted using a mortar and pestle and suspended in lysis buffer containing protease inhibitor cocktail (Roche, Basel, Switzerland). The proteins were quantified by the Bradford assay (Pierce/ThermoScientific) using bovine serum albumin as the standard. Total protein $(50 \mu \mathrm{g})$ was electrophoresed on $10 \%$ polyacrylamide gels and transferred onto nitrocellulose membranes (Bio-Rad Laboratories, Hercules, Calif). After blocking with 5\% fat-free milk solution, the membranes were exposed to monoclonal BMP-2 (1:1000; Abcam) primary antibody and a corresponding rabbit anti-mouse peroxidase-linked secondary antibody (1:5000; Cell Signaling Technology, Danvers, Mass). Blots were developed with ECL reagent and exposed on x-ray film. The films were scanned, and ImageJ software was used to quantitate the band densities.

\section{Statistical Analysis}

Univariate analyses were performed using the chi-square test for categorical variables and the $t$ test for continuous variables. The data are presented as the mean \pm standard deviation or standard error. A value of $P<.05$ (2-sided) was considered to be statistically significant.

\section{RESULTS \\ miRNA-141 Blocks TGF- $\beta$-Triggered VIC Response to Injury and Calcification}

To determine the effect of miRNA-141 on cellular activation and induction of calcification, porcine VICs were transfected with either a scrambled miRNA and stimulated with TGF- $\beta$ (scrambled miRNA plus TGF- $\beta$ ) or miRNA-141 and stimulated with TGF- $\beta$ (miRNA-141 plus TGF- $\beta$ ) and compared with nontransfected cells (control). The VICs were also transfected with scrambled miRNA only or miRNA141 only and demonstrated a phenotype similar to that of nontransfected controls (data not shown). TGF- $\beta$ triggered morphologic alterations, including transition from a spindle to stellate cell morphology and dense cellular clustering consistent with myofibroblastic transition in scrambled miRNA-transfected VICs. However, this phenotype was completely absent in miRNA-141-transfected VICs (Figure 1, A). TGF- $\beta$ further induced a synthetic osteoblastic phenotype in scrambled miRNA-transfected VICs characterized by calcification detected by alkaline phosphatase

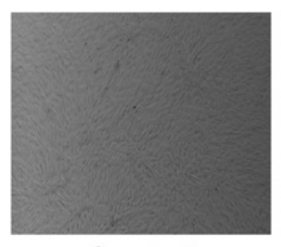

Control

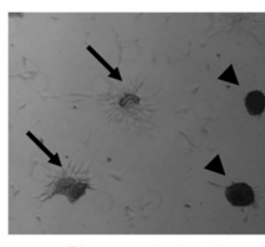

Scrambled miRNA + TGF $\beta$

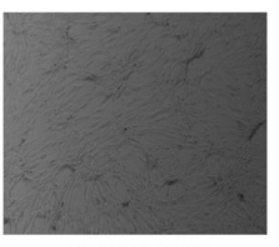

miRNA-141 + TGF $\beta$
A

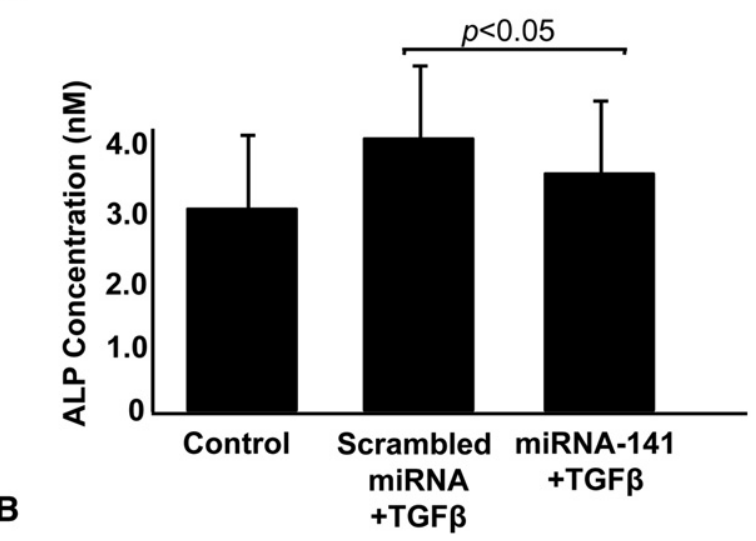

FIGURE 1. MicroRNA (miRNA)-141 represses valvular interstitial cell activation and osteoblastic transformation. Transforming growth factor$\beta(T G F-\beta)$ treatment induced (A) cellular aggregation (arrowheads) and stellate transformation (arrows), and (B) alkaline phosphatase (ALP) was repressed by miRNA-141 (mean \pm standard deviation; $n=5$ /group).

activity, which was also blocked by miRNA-141 overexpression $(4.0 \pm 0.21$ vs $3.55 \pm 0.18, P<.05$; Figure $1, B)$.

\section{miRNA-141 Blocks TGF- $\beta$-Triggered BMP-2 Signaling}

To determine the mechanism of miRNA-141 inhibition of VIC activation and calcification, the BMP-2 signaling pathway was studied. Exposure of VICs to TGF- $\beta$ resulted in increased transcripts of $B M P-2(1.4 \pm 0.1$ vs $1.0 \pm 0.1$, $P<.05$; Figure 2, A) and phosphorylation of downstream extracellular signal-regulated kinase 1/2 (Figure E1), which was partially reversed by overexpression of miRNA- 141 . We have further demonstrated increased transcript levels of downstream pro-osteogenic transcription factor RunX2 $(3.4 \pm 0.1$ vs $2.6 \pm 0.2, P<.05$; Figure $2, B)$. Increased RunX2 transcription levels were confirmed at protein levels after TGF- $\beta$ treatment in scrambled miRNA transfected and subsequent reversal in miRNA-141 overexpressing VICs (Figure E1).

\section{BAV and TAV Patient Characteristics}

The baseline patient characteristics are listed in Table 1. Overall, $58.3 \%$ of the patients were women, $77.8 \%$ had hypertension, $69.4 \%$ had hypercholesterolemia, and 33.3\% had diabetes mellitus. The BAV group was significantly 

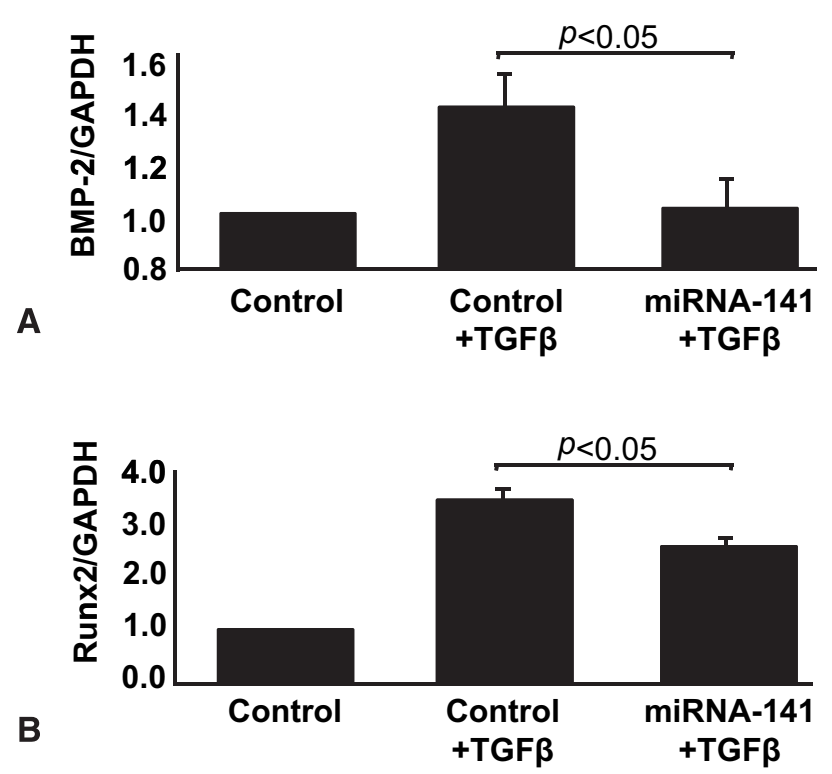

FIGURE 2. MicroRNA (miRNA)-141 represses bone morphogenetic protein-2 (BMP-2) signaling in cultured valvular interstitial cells (VICs). Transforming growth factor- $\beta(T G F-\beta)$ treatment induced (A) BMP-2 and (B) runt-related transcription factor-2 (RunX2) transcript expression compared with controls (mean \pm standard error; $\mathrm{n}=3$ /group). GADPH, Glyceraldehyde-3-phosphate dehydrogenase.

younger than the TAV group $(59.2 \pm 10.6$ years vs $77.4 \pm$ 6.2 years, $P<.0001$ ). No significant differences were found in the preoperative cardiac risk factors between the BAV and TAV patients. Of the BAV and TAV patients, $36.8 \%$ and

TABLE 1. Demographic, clinical, and echocardiographic data

\begin{tabular}{|c|c|c|c|}
\hline Variable & BAV & TAV & $P$ value \\
\hline Patients (n) & 19 & 17 & \\
\hline Age (yr) & $59.2 \pm 10.6$ & $77.4 \pm 6.2$ & $<.0001$ \\
\hline Women & $52.6(10)$ & $64.7(11)$ & .5 \\
\hline \multicolumn{4}{|l|}{ Risk factors } \\
\hline Hypertension & $73.7(14)$ & $82.4(14)$ & .7 \\
\hline Hypercholesterolemia & $57.9(11)$ & $82.4(14)$ & .2 \\
\hline Diabetes mellitus & $26.3(5)$ & $41.2(7)$ & .5 \\
\hline Chronic kidney disease & $5.3(1)$ & $11.8(2)$ & .6 \\
\hline Tobacco use & $47.4(9)$ & $23.5(4)$ & .2 \\
\hline \multicolumn{4}{|l|}{ Medications } \\
\hline Statin & $36.8(7)$ & $82.4(14)$ & .006 \\
\hline ACEi/ARB & $31.6(6)$ & $64.7(11)$ & .09 \\
\hline$\beta$-Blocker & $15.8(3)$ & $64.7(11)$ & .008 \\
\hline \multicolumn{4}{|l|}{ Echocardiographic parameters } \\
\hline $\operatorname{LVEF}(\%)$ & $56.8 \pm 7.3$ & $58.5 \pm 5.0$ & .5 \\
\hline Mean gradient $(\mathrm{mm} \mathrm{Hg})$ & $49.5 \pm 13.0$ & $46.5 \pm 20.7$ & 6 \\
\hline Aortic valve area $\left(\mathrm{cm}^{2}\right)$ & $0.8 \pm 0.2$ & $0.9 \pm 0.2$ & .7 \\
\hline Aortic insufficiency & $40(4)$ & $20(2)$ & 6 \\
\hline $\begin{array}{l}\text { Ascending aorta maximal } \\
\text { diameter }(\mathrm{cm})\end{array}$ & $4.2 \pm 0.7$ & $3.4 \pm 0.6$ & $<.001$ \\
\hline
\end{tabular}

Data presented as percentages, with numbers of patients in parentheses, unless otherwise noted. $B A V$, Bicuspid aortic valve; $T A V$, tricuspid aortic valve; $A C E i$, angiotensin-converting enzyme inhibitor; $A R B$, angiotensin receptor blocker; $L V E F$, left ventricular ejection fraction.
$82.4 \%$ were taking statin therapy $(P=.006)$ and $15.8 \%$ and $64.7 \%$ were taking $\beta$-blockers $(P=.008)$ preoperatively, respectively.

\section{Echocardiographic Measurement}

Preoperative transthoracic echocardiographic measurements, including left ventricular ejection fraction $(56.8 \%$ $\pm 7.3 \%$ vs $58.5 \% \pm 5.0 \%, P=.5$ ), aortic valve area $(0.8 \pm 0.2$ vs $0.9 \pm 0.2, P=.7)$, mean transvalvular gradient $(49.5 \pm 13.0$ vs $46.5 \pm 20.7 \mathrm{~mm} \mathrm{Hg}, P=.6)$, and the presence of any degree of aortic insufficiency $(40 \%$ vs $20 \%$, $P=.6$ ), were not different between the BAV and TAV patients (Table 1). Thus, both groups of patients were considered to have severe AS. The diameter of the ascending aorta was greater in the BAV patients than in the TAV patients $(4.2 \pm 0.7 \mathrm{~cm}$ vs $3.4 \pm 0.6 \mathrm{~cm} ; P<.001)$.

\section{Distinct miRNA Profiles in Human BAV and TAV}

Comparative global profile analysis of miRNA expression was performed using a microarray containing $1583 \mathrm{hu}-$ man miRNA probes. Two patients in each group were excluded at the microarray stage because of poor quality RNA and hybridization data. The global miRNA profiles were remarkably consistent and unique for bicuspid and tricuspid tissue $(\mathrm{n}=8$ patients/group; Figure $3, A)$. Microarray analysis demonstrated differential expression of 34 of 1583 miRNA sequences in BAV compared with TAV. Eight miRNAs were upregulated and 27 were downregulated (Tables E1 and E2). Expression of miRNA-141 was downregulated 14.5-fold in BAV compared with TAV leaflets by microarray. Confirmation with reverse transcriptasepolymerase chain reaction demonstrated a decrease in miRNA-141 expression in BAV compared with TAV patients $(5.6 \pm 1.7$ vs $8.2 \pm 1.5, P<.05$; Figure $3, B)$.

\section{BAV Leaflets Express Lower miRNA-14 and Greater BMP-2 Signaling}

We have demonstrated that miRNA-141 expression is associated with inhibition of BMP-2 signaling and calcification in vitro and that human BAV leaflets have lower miRNA-141 expression. Therefore, we sought to determine whether BMP-2 signaling is increased in BAV tissue compared with TAV tissue as a mechanistic explanation for early clinical stenosis in patients with bicuspid valves. Leaflets stained with von Kossa stain for calcification demonstrated profound calcification as a proportion of leaflet proper (Figure E2). Expression of $\alpha$-SMA is associated with a myofibroblastic phenotype in activated VICs. Immunohistochemistry for $\alpha$-SMA revealed positivity in close proximity to areas of calcification, suggesting a cellular source. Finally, BMP-2 staining revealed positivity in areas adjacent to $\alpha$-SMA and in close proximity to areas of valvular calcification in both bicuspid and tricuspid tissue (Figure E2). Image analysis and quantification of von Kossa 


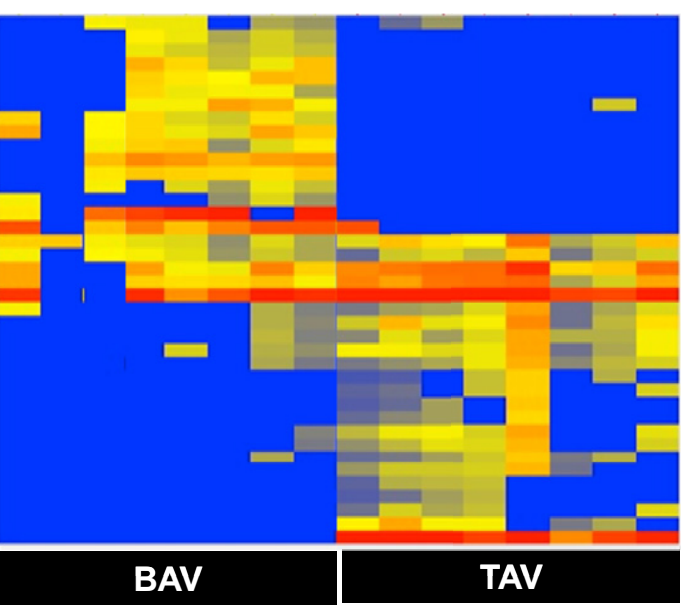

A
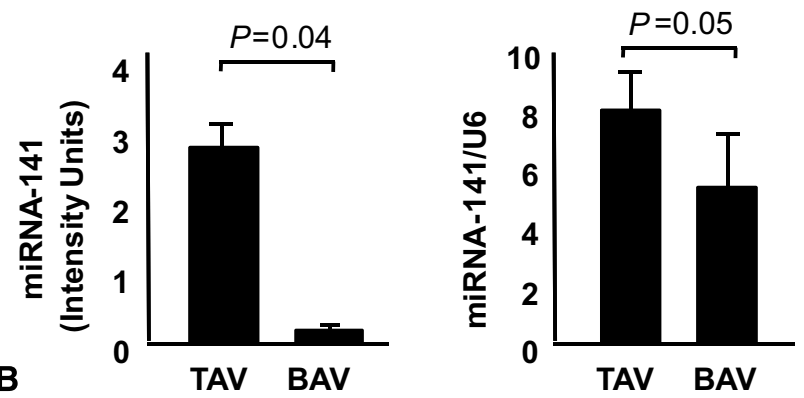

FIGURE 3. MicroRNA ( $m i R N A$ ) profile in human bicuspid aortic valve $(B A V)$ versus tricuspid aortic valve $(T A V)$ leaflets. A, Heat map showing the global profile of the relative levels of miRNA expression in BAVs and TAVs ( $\mathrm{n}=8$ /group). B, miRNA-141 is significantly repressed in BAV in microarray data (Left, mean \pm standard deviation; $n=8$ /group), which was also confirmed by reverse transcriptase polymerase chain reaction (Right; mean \pm standard error; $n=4$ /group).

staining demonstrated comparable calcification between bicuspid and tricuspid leaflets (Figure E3). Immunoblot analysis demonstrated that leaflet tissues from BAV expressed a modest but significant increase in BMP-2 compared with TAV leaflets, despite similar levels of calcification (Figure 4). Taken together, theses data suggest that activated myofibroblasts in BAV leaflets express greater levels of BMP-2, which might account for the early calcification seen clinically. Trends were seen toward greater expression of RunX2 and Dlx 5 in BAV leaflets on immunoblot analysis but this lacked statistical significance (data not shown).

\section{DISCUSSION}

In the present report, we have demonstrated that overexpression of miRNA-141 in cultured VICs blocks cellular activation and osteoblastic transformation, likely through a BMP-2-dependent mechanism. We then determined the miRNA profile of BAV and TAV leaflets, demonstrating relative downregulation of miRNA-141 in stenotic bicuspid
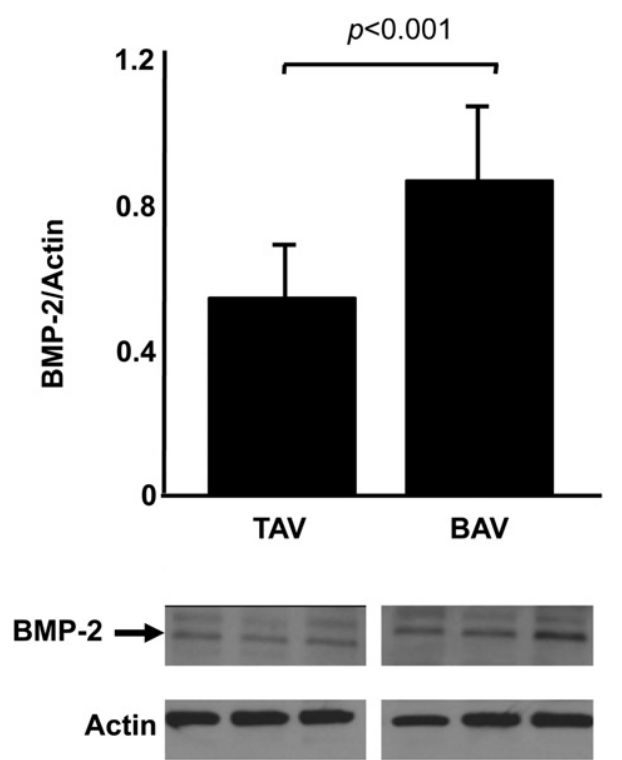

FIGURE 4. Calcified bicuspid aortic valve $(B A V)$ leaflets express greater bone morphogenetic protein-2 (BMP-2) compared with tricuspid aortic valve $(T A V)$ leaflets. BAV leaflets expressed greater BMP-2 expression than TAV leaflets and was found in close spatial association to areas of calcification (mean \pm standard deviation; $\mathrm{n}=10-12$ /group).

leaflets. This was associated with increased BMP-2 expression. These findings suggest that loss of miRNA-141 regulation of BMP-2-dependent calcification might underlie altered susceptibility toward calcific AS observed in patients with BAV.

Cellular signals driving aortic valve calcification are reminiscent of bone formation, and several lines of evidence suggest that BMP-2 is a central regulator of calcification in AS. Immunohistochemical staining has shown that stenotic human aortic valve leaflets express greater BMP-2 than nonstenotic leaflets. ${ }^{3}$ Treatment of cultured human and porcine VICs with BMP-2 triggered cellular aggregation and calcification. ${ }^{15}$ This likely occurs through extracellular signal-regulated kinase 1/2 and Smad signaling, with downstream expression of pro-osteogenic transcription factor RunX2 and the sialoprotein osteopontin. ${ }^{8}$ However, not all VICs are alike in their propensity to trigger BMP-2, because VICs from stenotic valves have greater BMP-2 than VICs from nonstenotic valves after stimulation with TollLike receptor-2/-4. ${ }^{16}$ Heterozygous Notch1 null mice develop BAV, and heterozygous Notchl mutations have been shown in 2 families with BAV disease and severe AS. ${ }^{17,18}$ Nigam and Srivastava ${ }^{17}$ have also demonstrated that Notch1 represses the BMP-2-dependent osteoblastic commitment of VICs, suggesting a possible mechanism for early calcification. In the present report, we have demonstrated that VICs from end-stage stenotic BAVs have increased BMP-2 signaling compared with stenotic TAVs and suggest that lower miRNA-141 might be an important upstream regulator. 
miRNA-141 is a member of the miRNA-200 family. In cancer models and bone formation, miRNA-141 is involved in regulation of cell fate decisions. ${ }^{11,19,20}$ In colorectal cancer, miRNA-141 is a critical regulator of the epithelial-to-mesenchymal transition, thereby inhibiting migration and cancer invasion. ${ }^{19,20}$ In bone, miRNA-141 regulates BMP-2-induced preosteoblastic differentiation by repression of distal-less homeobox $5 .{ }^{12}$ We have found that miRNA-141 expression represses the VIC response to injury simulated by TGF- $\beta$ exposure, a known stimulus for myofibroblast transformation. ${ }^{21}$ We have demonstrated cellular aggregation, transition to a stellate phenotype, and induction of calcification with TGF- $\beta$, which was completely reversed by miRNA-141. This is the first time that miRNA-141 has been shown to regulate aortic VIC activation. Our data are consistent with a role of miRNA-141 in regulation of cellular transition in multiple cell types. We believe that such regulation occurs in aortic VICs upstream of the BMP-2 pathway (Figure E4). However, the exact target of miRNA-141 in the BMP-2 pathway is unknown. A search of miRNA-141 target sequences within known BMP-2 signaling proteins using 2 separate miRNA target prediction programs (TargetScan, version 4.1 , available from www.targetscan.org; and miRBase Targets, version 5 , available from www.microrna.sanger.ac.uk/targets/v5/) did not reveal any matches.

We have demonstrated global downregulation of miRNA in stenotic bicuspid valves with greater than 3 times more genes significantly downregulated compared with TAVs. In the first report profiling miRNA in BAV compared patients with stenotic versus regurgitant phenotypes, 7 miRNAs were significantly different statistically. ${ }^{22}$ Specifically, miR-26a and miR-195 were reduced in the stenotic valves, and human VICs treated with miR-26a or miR$30 \mathrm{~b}$ mimics showed decreased mRNA levels of calcification-related genes, including BMP-2.That group compared stenotic and insufficient BAV, whereas we compared stenotic BAV and TAV. Surprisingly, no overlap was found in significantly altered miRNA in the 2 studies, which might have been due to the specific miRNA microarrays and different experimental groups used for comparison.

No medical treatment is available for AS, and the only definitive treatment is surgical replacement or transcatheter implantation. An improved understanding of the underlying pathogenetic mechanisms of valvular calcification could lead to the development of targeted treatments to inhibit stenosis development and delay or even avoid the need for valve replacement. A focused miRNA treatment of AS might take the form of antagomirs, complementary sequence oligonucleotides, which compete with the target mRNA to bind miRNA. More than 30 clinical trials targeting miRNA have been performed to date for a range of diseases, including heart failure, sepsis, melanoma, renal cell carcinoma, and infection by hepatitis $\mathrm{C}$ and human immunodeficiency virus. ${ }^{23}$ Considering the robustness of the role of miRNA-141 in cellular transformation and the large number of potential targets, any potential treatment would have to be carefully considered with regard to side effects, with the most worrisome being oncologic.

\section{Study Limitations}

The present study had some important limitations. The numbers of patients in the BAV and TAV groups were relatively small, and high-quality RNA was not obtained in approximately $33 \%$ of tissues, comparable to a recently published experience. ${ }^{24}$ Given that several known calcium signaling pathways exist and also that miRNAs might negatively regulate up to 200 target sequences, the overall significance of miRNA-141 regulation of BMP-2 remains to be determined. As a caveat, we found a lower use of statins by patients with BAV than by those with TAV $(36.8 \%$ vs $82.4 \%$ ). Statins have cholesterol-lowering and antiinflammatory properties. A recent meta-analysis has not demonstrated any effect of statins on AS progression; however, the possible effects of statins on miRNA-141 function are unknown. ${ }^{25}$ Finally, our studies of miRNA-141 regulation of BMP-2-dependent calcification have established an association but not causality. Additional work is needed to confirm our findings and to establish a causal relationship between miRNA-141 and calcification.

\section{CONCLUSIONS}

Our study has demonstrated that miRNA-141 represses the VIC response to injury and calcification in cultured porcine VICs. This occurs at least in part through BMP-2-dependent calcification. We have also shown that miRNA-141 is repressed in stenotic BAV compared with TAV leaflets and is associated with increased BMP-2 expression. This is the first study to demonstrate a potential regulatory role for miRNA-141 in calcific AS.

\section{References}

1. Fedak PW, Verma S, David TE, Leask RL, Weisel RD, Butany J. Clinical and pathophysiological implications of a bicuspid aortic valve. Circulation. 2002; 106:900-4.

2. Mohler ER III, Gannon F, Reynolds C, Zimmerman R, Keane MG, Kaplan FS Bone formation and inflammation in cardiac valves. Circulation. 2001;103 $1522-8$

3. Kaden JJ, Bickelhaupt S, Grobholz R, Vahl CF, Hagl S, Breukmann M, et al. Expression of bone sialoprotein and bone morphogenetic protein-2 in calcific aortic stenosis. J Heart Valve Dis. 2004;13:560-6.

4. Hogan BL. Bone morphogenetic proteins: multifunctional regulators of vertebrate development. Genes Dev. 1996;10:1580-94.

5. O'Brien KD, Kuusisto J, Reichenbach DD, Ferguson M, Giachelli C, Alpers CE, et al. Osteopontin is expressed in human aortic valvular lesions. Circulation. 1995;92:2163-8

6. Srivatsa SS, Harrity PJ, Maercklein PB, Kleppe L, Veinot J, Edwards WD, et al. Increased cellular expression of matrix proteins that regulate mineralization is associated with calcification of native human and porcine xenograft bioprosthetic heart valves. J Clin Invest. 1997;99:996-1009.

7. Wozney JM, Rosen V, Celeste AJ, Mitsock LM, Whitters MJ, Kritz RW, et al. Novel regulators of bone formation: molecular clones and activities. Science. 1988;242:1528-34 
8. Yang X, Meng X, Su X, Mauchley DC, Ao L, Cleveland JC Jr, et al. Bone morphogenic protein 2 induces RunX2 and osteopontin expression in human aortic valve interstitial cells: role of Smad1 and extracellular signal-regulated kinase 1/2. J Thorac Cardiovasc Surg. 2009;138:1008-15.

9. Lee MH, Kim YJ, Kim HJ, Park HD, Kang AR, Kyung HM, et al. BMP-2induced RunX2 expression is mediated by Dlx5, and TGF-beta 1 opposes the BMP-2-induced osteoblast differentiation by suppression of Dlx5 expression. $J$ Biol Chem. 2003;278:34387-94.

10. Ikeda T, Shirasawa T, Esaki Y, Yoshiki S, Hirokawa K. Osteopontin mRNA is expressed by smooth muscle-derived foam cells in human atherosclerotic lesions of the aorta. J Clin Invest. 1993;92:2814-20.

11. Filipowicz W, Bhattacharyya SN, Sonenberg N. Mechanisms of posttranscriptional regulation by microRNAs: are the answers in sight? Nat Rev Genet. 2008;9:102-14.

12. Itoh T, Nozawa Y, Akao Y. MicroRNA-141 and -200a are involved in bone morphogenetic protein-2-induced mouse pre-osteoblast differentiation by targeting distal-less homeobox 5. J Biol Chem. 2009;284:19272-9.

13. Ward C. Clinical significance of the bicuspid aortic valve. Heart. 2000;83:81-5.

14. Fayet C, Bendeck MP, Gotlieb AI. Cardiac valve interstitial cells secrete fibronectin and form fibrillar adhesions in response to injury. Cardiovasc Pathol. 2007;16:203-11.

15. Mohler ER III, Chawla MK, Chang AW, Vyavahare N, Levy RJ, Graham L, et al. Identification and characterization of calcifying valve cells from human and canine aortic valves. J Heart Valve Dis. 1999;8:254-60.

16. Yang X, Fullerton DA, Su X, Ao L, Cleveland JC Jr, Meng X, et al. Pro-osteogenic phenotype of human aortic valve interstitial cells is associated with higher levels of Toll-like receptors 2 and 4 and enhanced expression of bone morphogenetic protein 2. J Am Coll Cardiol. 2009;53:491-500.
17. Nigam V, Srivastava D. Notch1 represses osteogenic pathways in aortic valve cells. J Mol Cell Cardiol. 2009;47:828-34.

18. Garg V, Muth AN, Ransom JF, Schluterman MK, Barnes R, King IN, et al. Mutations in NOTCH1 cause aortic valve disease. Nature. 2005;437:270-4.

19. Hu M, Xia M, Chen X, Lin Z, Xu Y, Ma Y, et al. MicroRNA-141 regulates Smad interacting protein 1 (SIP1) and inhibits migration and invasion of colorectal cancer cells. Dig Dis Sci. 2010;55:2365-72.

20. Korpal M, Lee ES, Hu G, Kang Y. The miR-200 family inhibits epithelialmesenchymal transition and cancer cell migration by direct targeting of E-cadherin transcriptional repressors ZEB1 and ZEB2. J Biol Chem. 2008;283: 14910-4.

21. Walker GA, Masters KS, Shah DN, Anseth KS, Leinwand LA. Valvular myofibroblast activation by transforming growth factor-beta: implications for pathological extracellular matrix remodeling in heart valve disease. Circ Res. 2004; 95:253-60

22. Nigam V, Sievers HH, Jensen BC, Sier HA, Simpson PC, Srivastava D, et al. Altered microRNAs in bicuspid aortic valve: a comparison between stenotic and insufficient valves. J Heart Valve Dis. 2010;19:459-65.

23. Wahid F, Shehzad A, Khan T, Kim YY. MicroRNAs: synthesis, mechanism, function, and recent clinical trials. Biochim Biophys Acta. 2010;1803: 1231-43.

24. Moreno PR, Astudillo L, Elmariah S, Purushothaman KR, Purushothaman M, Lento PA, et al. Increased macrophage infiltration and neovascularization in congenital bicuspid aortic valve stenosis. J Thorac Cardiovasc Surg. 2011;142: 895-901.

25. Parolari A, Tremoli E, Cavallotti L, Trezzi M, Kassem S, Loardi C, et al. Do statins improve outcomes and delay the progression of non-rheumatic calcific aortic stenosis? Heart. 2011;97:523-9. 


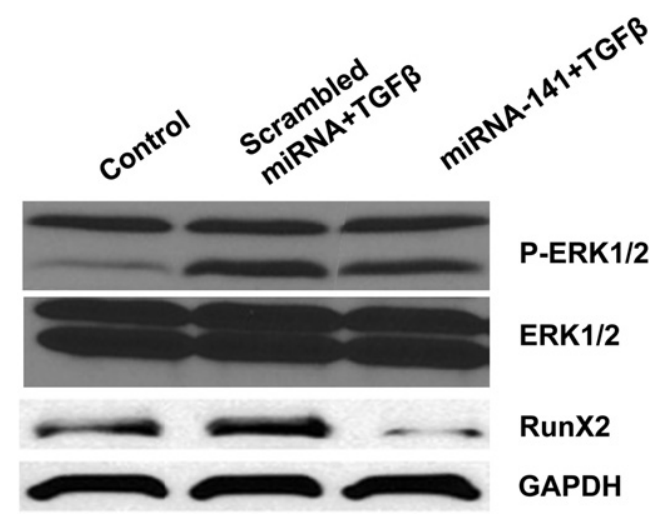

FIGURE E1. MicroRNA (miRNA)-141 represses transforming growth factor- $\beta$ (TGF- $\beta$ ) treatment-associated extracellular signal-regulated kinase $(E R K 1 / 2)$ activation and runt-related transcription factor-2 (RunX2) expression in cultured valvular interstitial cells $(\mathrm{n}=2$ /group). $P$, Phospho; $G A P D H$, glyceraldehyde-3-phosphate dehydrogenase.
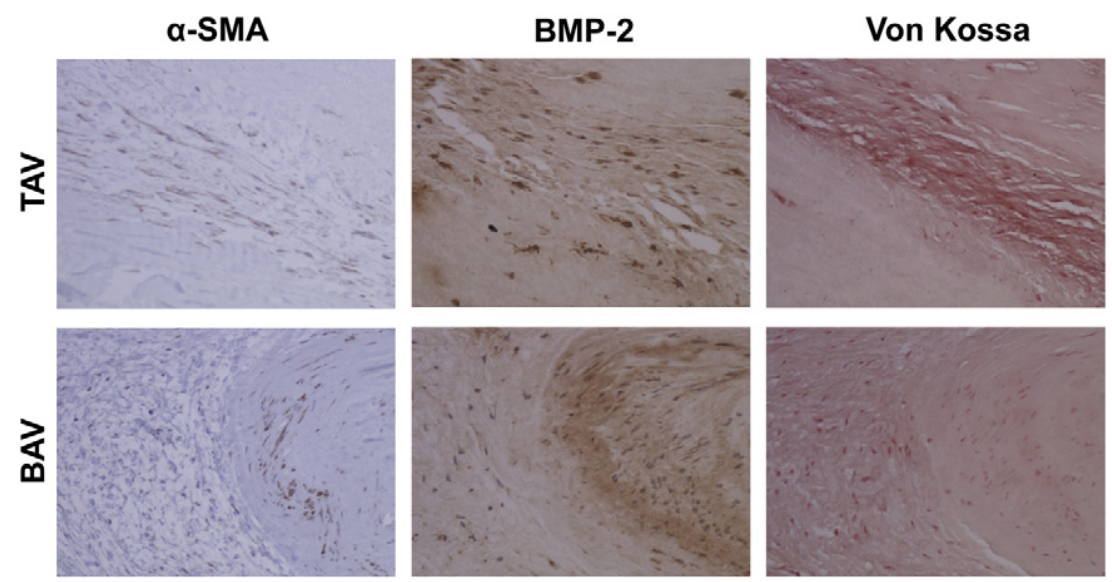

FIGURE E2. Calcified bicuspid aortic valve (BAV) leaflets co-express greater $\alpha$-smooth muscle actin $(\alpha-S M A)$ and bone morphogenetic protein-2 (BMP-2) positivity was in close spatial association to areas of calcification (Von Kossa stain; $n=10-12 /$ group). 


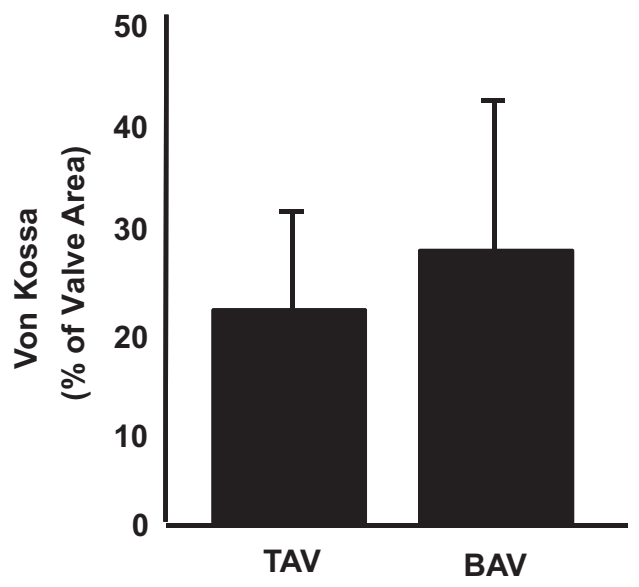

FIGURE E3. Bicuspid aortic valve $(B A V)$ and tricuspid aortic valve (TAV) leaflets expressed comparable levels of calcification $(\mathrm{n}=10-12 /$ group $)$.
TABLE E1. Upregulated miRNA expression in human BAV vs TAV

\begin{tabular}{lcc}
\hline \multicolumn{1}{c}{ miRNA } & Fold change (BAV/TAV) & $\boldsymbol{P}$ value \\
\hline hsa-miR-151-3p & 16.44 & .01 \\
hsa-miR-152 & 15.87 & .003 \\
hsa-miR-030e & 14.80 & .007 \\
hsa-miR-032 & 8.78 & .02 \\
hsa-miR-145 & 8.48 & .008 \\
hsa-miR-768-5p & 3.86 & .03 \\
hsa-miR-190 & 3.31 & .04 \\
hsa-miR-373* & 1.19 & .004 \\
\hline
\end{tabular}

miRNA, MicroRNA; $B A V$, bicuspid aortic valve; $T A V$, tricuspid aortic valve.

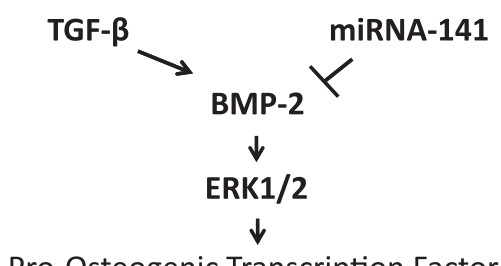

Pro-Osteogenic Transcription Factor:

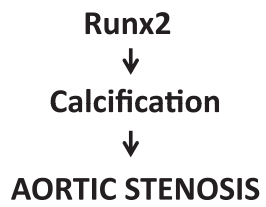

FIGURE E4. Proposed microRNA (miRNA)-141 regulation of bone morphogenetic protein-2 (BMP-2)-dependent calcification in aortic valve leaflets. Upstream activators of valvular interstitial cells, such as transforming growth factor- $\beta$ (TGF- $\beta$ ), trigger BMP-2 signaling and transcription of the pro-osteogenic transcription factor, runt-related transcription factor-2 (RunX2), leading to calcification and clinical aortic stenosis. miRNA-141 might be an important upstream regulator and, as such, the loss of expression such as in patients with bicuspid aortic valves $(B A V s)$, might beget dysregulated BMP-2-dependent calcification.
TABLE E2. Downregulated miRNA expression in human BAV vs TAV

\begin{tabular}{|c|c|c|}
\hline miRNA & Fold change (BAV/TAV) & $P$ value \\
\hline hsa-miR-370 & 0.83 & .020 \\
\hline hsa-miR-022 & 0.56 & .04 \\
\hline hsa-miR-1972 & 0.54 & .04 \\
\hline hsa-miR-330-5p & 0.47 & .04 \\
\hline hsa-miR-566-pre & 0.39 & .05 \\
\hline hsa-miR-1469 & 0.38 & .03 \\
\hline hsa-miR-1908 & 0.30 & .01 \\
\hline hsa-miR-648-pre & 0.23 & .01 \\
\hline hsa-miR-637 & 0.23 & .01 \\
\hline hsa-miR-1282 & 0.23 & .004 \\
\hline hsa-miR-622 & 0.18 & .05 \\
\hline hsa-miR-486-3p & 0.15 & .04 \\
\hline hsa-miR-187 & 0.14 & .05 \\
\hline hsa-miR-1909* & 0.12 & .03 \\
\hline hsa-miR-564 & 0.08 & .01 \\
\hline hsa-miR-141 & 0.07 & .04 \\
\hline hsa-miR-027a & 0.07 & .009 \\
\hline hsa-miR-194* & 0.05 & .01 \\
\hline hsa-miR-125b-1-pre & 0.00 & .003 \\
\hline hsa-miR-551a-pre & 0.00 & .03 \\
\hline hsa-miR-575 & 0.00 & .03 \\
\hline hsa-miR-585-pre & 0.00 & .03 \\
\hline hsa-miR-211-pre & 0.00 & .05 \\
\hline hsa-miR-449b* & 0.00 & .05 \\
\hline hsa-miR-124-3-pre & 0.00 & .05 \\
\hline hsa-miR-185-pre & 0.00 & .05 \\
\hline hsa-miR-1202 & 0.00 & .05 \\
\hline
\end{tabular}

miRNA, MicroRNA; $B A V$, bicuspid aortic valve; $T A V$, tricuspid aortic valve. 